\title{
Optical Properties and Density Functional Theory Study of Single Crystal Glycine
}

\author{
P. Kavitha, J. Shanthi, and P. R. Deepthi
}

\begin{abstract}
Single crystals of L-Glycine were grown from aqueous solution by slow evaporation method. The optical properties of the grown crystals were studied using UV-Vis spectral analysis. Optical absorption study reveals the transparency of the crystal in the entire visible region and the cutoff wavelength was found to be $250 \mathrm{~nm}$. Powder X-ray diffraction analysis confirms the crystalline nature of grown L-Glycine. The value of chemical hardness at B3LYP/6-31G level of theory was found to be 3.52 .
\end{abstract}

Index Terms-Glycine, solution method, UV-visible spectrometry, XRD, FTIR.

\section{INTRODUCTION}

Over the recent years extensive investigations have been made on the amino acid crystals by researchers. Among organic crystals for NLO applications, amino acids display specific features of interest such as (i) molecular chirality, which secures acentric crystallographic structure, (ii) absence of strongly conjugated bonds, leading to wide transparency ranges in the visible and UV spectral regions, (iii) zwitterionic nature of the molecule, which favours crystal hardness [1]-[3]. Glycine is the simplest of all amino acids; it has no asymmetric carbon and in the present study Glycine has been grown by slow evaporation method and the grown crystals was subjected to UV-Visible spectrometry, $\mathrm{X}$-Ray diffraction analysis and geometrical parameter measurement using Density Functional theory

\section{MATERIALS AND METHODS}

Single crystals of pure L-Glycine were grown by dissolving Analytical Grade L-Glycine (Amino acetic acid, $\mathrm{NH}_{2} \mathrm{CH}_{2} \mathrm{COOH}$ ) in double distilled water and stirred well for about two hours to get saturation solution. The resulting solution was filtered and then placed in a water bath maintained at constant temperature $\left(35^{\circ} \mathrm{C}\right)$. The solution was then allowed to evaporate in a petri dish to obtain seed crystals. Defect free seed crystal was suspended in the mother solution, which was allowed to evaporate at ambient temperature. Optically good quality single crystals were obtained within a week.

Manuscript received April 17, 2012; revised May 29, 2012.

P. Kavitha is with the Research scholar, Department of Physics, Avinashilingam University for women Coimbatore, Tamilnadu, India 641038

J. Shanthi is with the Department of Physics, Avinashilingam University for women, Coimbatore, Tamilnadu, India - 641038

P. R. Deepthi is with the Oxford College Of Engineering, Bangalore-560068

\section{RESULTS AND DISCUSSION}

\section{A. Optical Studies}

The UV-Vis-NIR spectrum of crystal is shown in Fig1, the absorption of UV and visible light involves the promotion of electrons in $\sigma$ and л orbital from the ground state to higher energy states. Wide transmission spectrum is very important for any NLO material and it can be of practical use only if it has wide transparency window [4]. The UV cutoff for the grown crystal was found to be $250 \mathrm{~nm}$ and low absorption through the entire visible region is one of the most desired properties for the fabrication of optoelectronic devices.

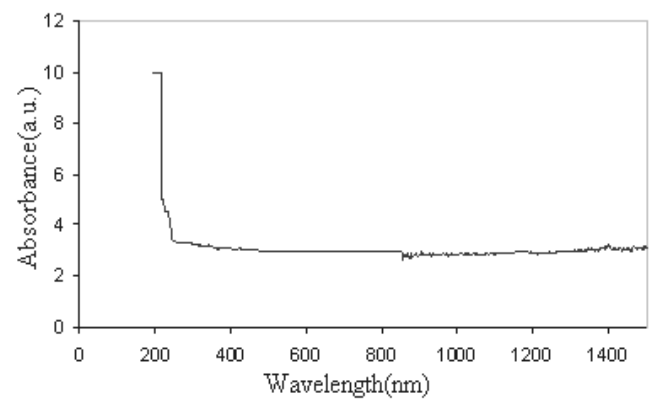

Fig. 1. Optical absorbance spectra of L-Glycine

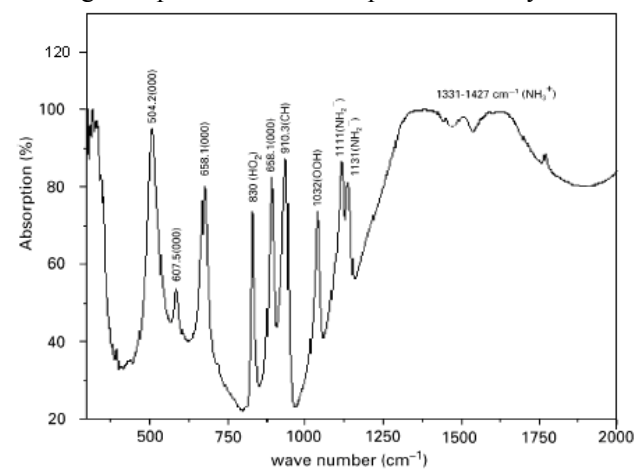

Fig. 2. IR spectra of L-GLYCINE crystal

\section{B. Infrared Spectra}

The Infrared spectral analysis is effectively used to understand the chemical bonding and it provides information about molecular structure of the synthesized compound. Fourier transform infrared (FTIR) was recorded in the range $400-2000 \mathrm{~cm}^{-1}$ using the Perkin Elmer grating infrared spectrophotometer. The sample used was in pellet form mixed with $\mathrm{KBr}$. The characteristic absorption peaks are observed in the range from $400-2000 \mathrm{~cm}^{-1}$ IR and is shown in Fig 2.

The absorption peaks due to carboxylate group are observed at 504.2, 607.5 and $658.1 \mathrm{~cm}^{-1}$, respectively. Similarly the absorption peaks due $\mathrm{NH}^{3+}$ group are observed 
at 1111,1131 and $1501 \mathrm{~cm}^{-1}$, respectively. The presence of $\mathrm{NO}_{3}^{-}$group is conformed due to absorption peak at $858 \mathrm{~cm}^{-1}$.Peak at 1032,1410 and $1613 \mathrm{~cm}^{-1}$ are attributed to $\mathrm{CCN}, \mathrm{COO}^{-}$and $\mathrm{CH}_{2}$ groups respectively. Others bands of $\mathrm{COO}^{-}$mode deformation were shown at 658.1, 607.5 and $504.2 \mathrm{~cm}^{-1}$, in the spectrum. These results confirmed that Glycine molecules exist in zwitterions form inside the grown L-Glycine crystal [5].

\section{Powder X-Ray Diffraction}

$\mathrm{X}$-Ray powder diffraction analysis was carried out for the identification of the synthesized crystal. Powder XRD pattern was obtained using PANALYTICAL X-Ray diffractrometer with a $\mathrm{CuK} \alpha$ radiation. The sample was scanned over the range $20^{\circ}-70^{\circ}$. The XRD pattern of L-Glycine is shown in Fig 3. The crystallite size was calculated using Scherrer equation.

$$
\mathrm{t}=\mathrm{K} \lambda / \beta \cos \theta
$$

where $\mathrm{k}$ is the scherrer constant, $\lambda$ is the wavelength of the $\mathrm{x}$-ray, and $\beta$ is the integral breadth of reflections (in radian). The relatively low FWHM value of main peak observed at the $2 \theta$ position of $71.24^{\circ}$ shows that crystalline perfection is reasonably good. Measured XRD parameters are presented in Table. I.

TABLE I: Computed VAlues of CRystallite Size

\begin{tabular}{|c|c|c|c|}
\hline S.NO & POS $\left[{ }^{\circ} 2 \mathrm{Th}\right]$ & FWHM & $\mathrm{P}=\mathrm{K} \lambda / \beta \operatorname{Cos} \theta$ \\
\hline 1. & 14.5560 & 0.3016 & 4.7497 \\
\hline 2. & 57.5790 & 0.7083 & 3.65123 \\
\hline 3. & 61.3927 & 0.1476 & 19.6195 \\
\hline 4. & 71.2497 & 0.1166 & 36.9937 \\
\hline 5. & 78.9782 & 0.2036 & 35.6210 \\
\hline
\end{tabular}

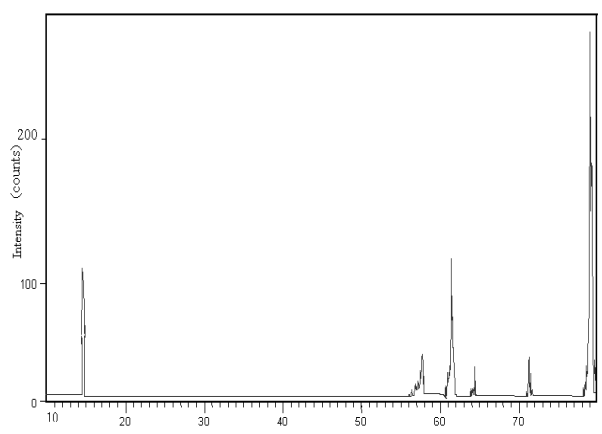

Fig. 3. Powder XRD pattern of L-Glycine

\section{Geometrical Parameters}

Molecular geometries can be specified in terms of bond length and bond angle. The geometrical parameters were calculated for Glycine using B3LYP/6-31G of density functional theory.

The Bond Length and Bond angle

The bond length is defined to be the average distance between the centers of two atoms bonded together in any given molecule. A bond angle is the angle formed between Three atoms across at least two bonds. The bond length of
$\mathrm{N}-\mathrm{H}$ obtained by density functional theory is $1.019 \AA$ and the bond length of $\mathrm{C}-\mathrm{H}$ is $1.099 \AA$. Similarly using DFT theory the Hydroxyl bond length is found to be 0.972 , which is shown in the Table II. The bond angles of H-N-H, H-N-C, $\mathrm{N}-\mathrm{C}-\mathrm{H}, \quad \mathrm{C}-\mathrm{C}=\mathrm{O}$ at density functional theory 104.644 , $108.705,109.243,123.863$ respectively.

TABLE II: BOND LENGTH $(\AA)$, BOND ANGLE ( $)$ OF GLYCINE OPTIMIZED AT B3LYP/6-31G

\begin{tabular}{|c|c|}
\hline \multicolumn{2}{|c|}{ BO B3LYP/6-31G } \\
\hline N-H & 1.019 \\
\hline C-H & 1.099 \\
\hline C-C & 1.535 \\
\hline C-O & 1.360 \\
\hline C=O & 1.204 \\
\hline O-H & 0.972 \\
\hline C-N & 1.450 \\
\hline \multicolumn{2}{|c|}{ BOND ANGLE } \\
\hline H-N-H & 104.644 \\
\hline H-N-C & 108.705 \\
\hline N-C-H & 109.243 \\
\hline H-C-H & 106.006 \\
\hline H-C-C $-\mathrm{C}=\mathrm{O}$ & 108.376 \\
\hline C-C-O & 123.863 \\
\hline C-O-H & 115.618 \\
\hline
\end{tabular}

Chemical Potential and Chemical Hardness

Chemical potential and chemical hardness are important tools to study the stability of the molecular system, which also have been calculated for the Glycine and are given in Table 3. These two parameters of a molecular system are the first and second derivative of energy with respect to the number of electrons. The chemical potential of Glycine is found as -3.36. The value of chemical hardness at B3LYP/6-31G level of theory is found to be 3.52 and are shown in the Table III

Dipole moment and Energy

The dipole moment is the first derivative of energy with respect to an applied electric field. The dipole moment $(\mu)$ for Glycine is 2.88 at B3LYP/6-31G level of theory.

\begin{tabular}{|c|c|}
\hline PARAMETERS & B3LYP/6-31G \\
\hline Energy(Hartree) & -284.41 \\
\hline Dipole moment & 2.88 \\
\hline Chemical potential $(\mu)$ & -3.36 \\
\hline Chemical hardness $(\eta)$ & 3.52 \\
\hline
\end{tabular}

\section{CONCLUSION}

Optically good quality crystals of L-Glycine have been grown by slow evaporation method. UV-Vis spectra showed that L-Glycine was optically transparent through 1200-350nm and hence suggests the suitability of this material for SHG applications of IR and diode laser. Powder $\mathrm{X}$-ray diffraction analysis confirms the crystalline nature of grown L-Glycine. The geometrical parameters such as bond length, bond angle, chemical potential, chemical hardness, 
dipole moment and energy of the Glycine molecule were calculated using density functional methods. The energy shows that the most stable structure of glycine is observed by density functional theory method.

\section{REFERENCES}

[1] M. D. Aggarwal, J. Stephens, A. K. Batra, and R. B. Lal, "Bulk crystal growth and characterization of semiorganic Nonlinear optical materials," J. Optoelectronics Adv Mater, vol. 5, pp. 555, 2003.

[2] C. Razzetti, M. Ardoino, L. Zanotti, M. Zha, and C. Paorici, "Solution Growth and Characterisation of L-Alanine Single Crystals," Cryst. Research Technol, vol. 37, pp. 456-465, May 2002.
[3] S. S. Raman and A. M. P, Kaliyamurthyanand, "Growth, Structural, Optical, Mechanical and Dielectric Properties of Glycine Sodium Nitrate (Gsn) Single Crystal," J. Optoelectronics and Biomedical Materials, vol. 1, pp. 129-139, July 2010.

[4] T. Mallik and T. Kar, "Optical Thermal and Structural Characterization of an Nlo Crystal, L-Arginine Perchlorate," J. Cryst. Growth, vol. 274, pp. 251-255, Jan 2005.

[5] J. H. Paredes, D. G. Mitnik, H. E. E. Ponce, M. E. A. Ramos, and A. D. Moller, "Band structure, optical properties and infrared spectrum of glycine-sodium nitrate crystal," J. Mol strict, vol. 875, pp. 295-301, March 2008. 